\title{
Determination of the optimum operating time for batch isothermal performance of enzyme-catalyzed multisubstrate reactions
}

\author{
F. X. Malcata
}

$\begin{array}{lll}\begin{array}{l}\text { Abstract This communication consists of a mathematical } \\ \text { analysis encompassing the maximization of the average rate of }\end{array} & k_{i} & \mathrm{I} / \mathrm{s} \\ \text { monomer production in a batch reactor performing an } & & \\ \text { enzymatic reaction in a system consisting of a multiplicity } & k_{c a t, i} i & 1 / \mathrm{s} \\ \text { of polymeric substrates which compete with one another for } & & \mathrm{mol} / \mathrm{m}^{3} \\ \text { the active site of a soluble enzyme, under the assumption that } & K_{m} & - \\ \text { the form of the rate expression is consistent with the } & L & - \\ \begin{array}{l}\text { Michaelis-Menten mechanism. The general form for the } \\ \text { functional dependence of the various substrate concentrations }\end{array} & M_{i} & - \\ \begin{array}{l}\text { on time is obtained in dimensionless form using matrix } \\ \text { terminology; the optimum batch time is found for a simpler }\end{array} & r_{1} & \mathrm{~mol} / \mathrm{m}^{3} \mathrm{~s} \\ \text { situation and the effect of various process and system variables } & r_{i} & \mathrm{~mol} / \mathrm{m}^{3} \mathrm{~s} \\ \text { thereon is discussed. The reasoning developed here emphasizes, } & r_{o p t}^{*} & - \\ \text { in a quantitative fashion, the fact that the commonly used } & & \\ \text { lumped substrate approaches lead to nonconservative decisions } & \mathrm{S} & - \\ \text { in industrial practice, and hence should be avoided when } & \mathrm{S}_{1} & - \\ \text { searching for trustworthy estimates of optimum operation. } & \mathrm{S}_{i} & -\end{array}$

$\begin{array}{ll}\begin{array}{l}\text { List of } \\ \mathbf{0}\end{array} & \mathrm{symbols} \\ \boldsymbol{a} & 1 / \mathrm{s} \\ \boldsymbol{A} & 1 / \mathrm{s} \\ \boldsymbol{b} & 1 / \mathrm{s} \\ C & \mathrm{~mol} / \mathrm{m}^{3} \\ \boldsymbol{C} & \mathrm{mol} / \mathrm{m}^{3} \\ \boldsymbol{C}_{0} & \mathrm{~mol} / \mathrm{m}^{3} \\ \boldsymbol{C}_{-01} & \mathrm{~mol} / \mathrm{m}^{3} \\ C_{E, \text { tot }} & \mathrm{mol} / \mathrm{m}^{3} \\ C_{i} & \mathrm{~mol} / \mathrm{m}^{3} \\ C_{i, o} & \mathrm{~mol} / \mathrm{m}^{3} \\ \text { E } & - \\ \boldsymbol{I} & - \\ \boldsymbol{K} & 1 / \mathrm{s}\end{array}$

row vector of zeros

row vector of rate constants

$k_{i}(i=2, \ldots, N)$

matrix of rate constants $k_{i}$ and $t_{\text {lag }} \quad \mathrm{s}$

$k_{-i}(i=2, \ldots, N)$

row vector of rate constant $k_{2}$ and zeros

molar concentration of $S$

vector of molar concentrations of

$C_{i}(i=0,1,2, \ldots, N)$

column vector of initial molar

concentrations of $C_{i}(i=0,1,2, \ldots, N)$

column vector of initial molar

concentrations of $C_{i}(i=2, \ldots, N)$

total molar concentration of enzyme molecules

molar concentration of

$S_{i}(i=0,1,2, \ldots, N)$

initial molar concentration of

$S_{i}(i=0,1,2, \ldots, N)$

enzyme molecule

identity matrix

matrix of lumped rate constants

1

\section{Mathematical analysis}

F. X. Malcata

Escola Superior de Biotecnologia, Universidade Católica Portuguesa, Rua Dr. António Bernardino de Almeida, 4200 Porto, Portugal pseudo-first order lumped rate constant associated with the formation of $S_{i-1}(i=1,2, \ldots, N)$

first order rate constant associated with the formation of $S_{i-1}(i=1,2, \ldots, N)$

Michaelis-Menten constant number of distinct eigenvalues multiplicity of the $i$-th eigenvalue maximum number of monomer residues in a single polymeric molecule rate of formation of $S_{0}$ rate of release of $S_{i-1}$ maximum average dimensionless rate of production of monomer $S_{0}$ lumped, pseudo substrate inert moiety substrate containing $i$ monomer residues, each labile to detachment as $S_{0}$ by enzymatic action

$(i=1,2, \ldots, N)$

time elapsed since startup of batch reaction time interval required for cleaning, loading, and unloading the batch reactor time interval leading to the maximum average rate of monomer production eigenvectors associated with eigenvalue $\lambda_{i}\left(i=1,2, \ldots, L ; j=1,2, \ldots, M_{i}\right)$

Greek symbols

$\alpha_{i j} \quad \mathrm{~mol} / \mathrm{m}^{3} \quad$ arbitrary constant associated with eigenvalue $\lambda_{i}(i=1,2, \ldots, L$; $j=1,2, \ldots, M_{i}$ ) generic eigenvalue $i$-th eigenvalue

Enzymes are the biological catalysts of nature. These catalytically active globular proteins possess, over the inorganic (or synthetic) catalysts, the advantages of extremely high activity, selectivity, and controllability [1]. Due to their in vivo requirements, some degradative enzymes show, nevertheless, considerable affinity to a wide variety of polymeric substrates provided that these substrates share a common type of labile covalent bond [2]. In this situation, the various reactants 
compete with each other for the active site of the enzyme irrespective of their sequence of monomer residues or overall molecular weight. Examples documented in the literature include the action of such hydrolases as lysozyme on mucopolysaccharides of bacterial cell walls [3], and amyloglucosidase on amylose [4, 5].

Of particular interest here are the reactions effected by soluble exo-hydrolases (i.e. enzymes that cleave ester, glycosidic or peptide bonds next to the ends of polymeric carbon backbones, thus releasing monomeric subunits) which are not catalytically active on dimeric or monomeric subunits (e.g. exoproteases deprived of dipeptidolytic activity) on complex aqueous mixtures of substrates consisting of linear biopolymers of various chain lengths. The general reaction mechanism can be represented as follows:

$$
\mathrm{S}_{1}-\mathrm{S}_{i}+\mathrm{E} \stackrel{K_{m}}{\rightleftarrows} \mathrm{E}\left(\mathrm{S}_{1}-\mathrm{S}_{i}\right)
$$$$
\stackrel{k_{c a t i i+1}}{\longrightarrow} \mathrm{E}+\mathrm{S}_{1}-\mathrm{S}_{i-1}+\mathrm{S}_{0}, \quad i=1,2, \ldots, N-1
$$

$\mathrm{S}_{1}-\mathrm{S}_{1}+\mathrm{E} \stackrel{K_{m}}{\rightleftarrows} \mathrm{E}\left(\mathrm{S}_{1}-\mathrm{S}_{1}\right) \stackrel{k_{\text {caut } 2}}{\longrightarrow} \mathrm{E}+\mathrm{S}_{1}+\mathrm{S}_{0}$

where $S_{1}-S_{i}$ denotes a substrate made of $i$ detachable monomeric subunits (i.e. $\mathrm{S}_{0}$ ) out of a total of $i+1$ subunits, $\mathrm{S}_{1}$ denotes an inert moiety, $\mathrm{E}$ denotes an enzyme molecule, $N$ is the maximum number of subunits in a single polymeric molecule, $K_{m}$ is the equilibrium constant associated with the dissociation of every type of enzyme/substrate complex (traditionally known as Michaelis-Menten constant), and $k_{c a t, i}$ is the first order rate constant associated with the formation of substrate containing $i-1$ total monomeric subunits. Assuming that (a) $S_{0}$ does not bind to the enzyme, (b) $S_{1}$ can bind to the enzyme but can not be transformed by it, and (c) $S_{1}-S_{i}$ $(i=1,2, \ldots, N)$ binds to the enzyme and can be transformed by it, the rate expression associated with each one of the above enzyme-catalyzed reactions can be written as [6]

$r_{i}=\frac{k_{c a t, i} C_{E, \text { tot }} C_{i}}{K_{m}+\sum_{j=1}^{N} C_{j}}, \quad i=2,3, \ldots, N$

where $r_{i}(i=1,2, \ldots, N-1)$ denotes the rate of the $i$-th reaction (i.e. the rate of consumption of substrate $S_{1}-S_{i}$, or, equivalently, $\left.S_{i+1}\right), C_{i}$ the molar concentration of substrate $S_{i}$, and $C_{E, \text { tot }}$ the total concentration of enzyme molecules. The rate of formation of $S_{0}$ is therefore given by

$r_{1}=\frac{\sum_{i=2}^{N} k_{c a t, i} C_{E, \text { tot }} C_{i}}{K_{m}+\sum_{j=1}^{N} C_{j}}$.

The chemical reaction is assumed to be carried out in a batch stirred reactor under isothermal conditions and absence of enzyme deactivation. Under these conditions, Eqs. (2) and (3) allow one to write the mass balance to the set of $N$ species of form $S_{i}(i=0,1, \ldots, N)$ in the following condensed fashion:

$\frac{\mathrm{d} C}{\mathrm{~d} t}=\boldsymbol{K} \boldsymbol{C}$,

$t=0, \quad C=C_{0}$.

where $C \equiv\left(\begin{array}{lllllll}C_{0} & C_{1} & C_{2} & \ldots & C_{N}\end{array}\right)^{T}, \boldsymbol{C}_{0} \equiv\left(\begin{array}{lllll}C_{0,0} & C_{1,0} & C_{2,0} & \ldots & C_{N, 0}\end{array}\right)^{T}$, matrix $\boldsymbol{K}$ is defined as

$K \equiv$

$\left[\begin{array}{ccccccccc}0 & 0 & k_{2} & k_{3} & k_{4} & \ldots & k_{N-2} & k_{N-1} & k_{N} \\ 0 & 0 & k_{2} & 0 & 0 & \ldots & 0 & 0 & 0 \\ 0 & 0 & -k_{2} & k_{3} & 0 & \ldots & 0 & 0 & 0 \\ 0 & 0 & 0 & -k_{3} & k_{4} & \ldots & 0 & 0 & 0 \\ 0 & 0 & 0 & 0 & -k_{4} & \ldots & 0 & 0 & 0 \\ \ldots & \ldots & \ldots & \ldots & \ldots & \ldots & \ldots \ldots \ldots & \ldots & \ldots \\ 0 & 0 & 0 & 0 & 0 & \ldots & 0 & 0 & 0 \\ 0 & 0 & 0 & 0 & 0 & \ldots & k_{N-2} & 0 & 0 \\ 0 & 0 & 0 & 0 & 0 & \ldots & -k_{N-2} & k_{N-1} & 0 \\ 0 & 0 & 0 & 0 & 0 & \ldots & & -k_{N-1} & k_{N} \\ 0 & 0 & 0 & 0 & 0 & \ldots & 0 & 0 & -k_{N}\end{array}\right]$,

and $t$ is the time elapsed since startup of the batch reactor. The definition of the lumped rate constants is as follows:

$k_{i}=\frac{k_{\text {cat }, i} C_{E, \text { tot }}}{K_{m}+\sum_{j=1}^{N} C_{j}}=\frac{k_{\text {cat }, i} C_{E, \text { tot }}}{K_{m}+\sum_{j=1}^{N} C_{j, 0}}, \quad i=2,3, \ldots, N$.

Equation (4) may be rearranged via partition into submatrices, viz.

$$
\begin{aligned}
& \frac{\mathrm{d}\left(\begin{array}{c}
C_{0} \\
C_{1} \\
\boldsymbol{C}_{-01}
\end{array}\right)}{\mathrm{d} t}=\left(\begin{array}{lll}
0 & 0 & \boldsymbol{a} \\
0 & 0 & \boldsymbol{b} \\
0 & 0 & \boldsymbol{A}
\end{array}\right)\left(\begin{array}{c}
C_{0} \\
C_{1} \\
\boldsymbol{C}_{-01}
\end{array}\right) \\
& t=0,\left(\begin{array}{c}
C_{0} \\
C_{1} \\
\boldsymbol{C}_{-01}
\end{array}\right)=\left(\begin{array}{c}
C_{0,0} \\
C_{1,0} \\
C_{-01,0}
\end{array}\right)
\end{aligned}
$$

where $C_{-01}$ is the $(N-1)$-th order column vector defined as

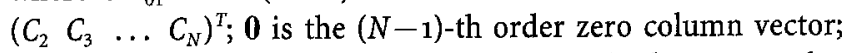
$a$ is the $(N-1)$-th order row vector with generic element $a_{i} \equiv k_{i+1}$ for $1 \leqslant i \leqslant N-1 ; \boldsymbol{b}$ is the $(N-1)$-th order row vector with generic element $a_{i} \equiv k_{i+1}$ for $i=1$ and $a_{i} \equiv 0$ for $2 \leqslant i \leqslant N-1 ; \boldsymbol{A}$ is the $(N-1)$-th order square band matrix of generic element $A_{i j} \equiv-k_{i+1}$ for $j=i, A_{i j} \equiv k_{i+2}$ for $j=i+1$, and $A_{i j} \equiv 0$ for $j \neq \mathrm{i}$, $\mathrm{i}+1$; and $C_{i, 0}$ denotes the initial concentration of substrate $S_{i}$.

In the most general situation, the solution of Eq. (7) is given by

$$
\begin{gathered}
C_{0}=C_{0,0}+\sum_{i=2}^{N} k_{i} \int_{0}^{t} C_{i} \mathrm{~d} t, \\
C_{1}=C_{1,0}+\sum_{i=2}^{N}\left(C_{i, 0}-C_{i}\right), \\
C_{-01}=\sum_{i=1}^{L}\left(\sum_{j=0}^{M_{i}-1} \alpha_{i j} \frac{t^{j}}{j !}\left(A-\lambda_{i} I\right)^{j} v_{i j}\right) \exp \left\{-k_{i} t\right\}, \\
t=0, \quad C_{-01}=C_{-01,0}
\end{gathered}
$$

where $M_{i}$ is the multiplicity of $\lambda_{i}, L$ is the number of distinct values $\lambda_{i}, \alpha_{i j}\left(i=1,2, \ldots, L ; j=1,2, \ldots, M_{i}\right)$ are arbitrary integration constants associated with $\lambda_{i}$, and $I$ is the $(N-1)$-th order identity matrix; as expected, $\sum_{i=1}^{L} M_{i}=N-1$. The $\lambda_{i}$ are the 
eigenvalues of matrix $A$, i.e. the $\lambda$ values which satisfy the condition

$$
|A-\lambda I|=0 \text {. }
$$

Due to the upper triangular nature of $A$, the eigenvalues $\lambda_{1}, \lambda_{2}, \ldots, \lambda_{L}$ are simply the distinct solutions of equation $\operatorname{tr}(A-\lambda I)=0$, where tr denotes the product of all elements located on the main diagonal of the matrix in question. The $\boldsymbol{v}_{i j}\left(i=1,2, \ldots, L ; j=1,2, \ldots, M_{i}\right)$ denote the $M_{i}$ linearly independent $(N-1)$-th order eigenvectors of $\boldsymbol{A}$ associated with every eigenvalue $\lambda=-k_{i}(i=1,2, \ldots, L)$ of multiplicity $M_{i}$ [7]. Such eigenvectors can be obtained as nontrivial solutions of

$\left(A+k_{i} I\right)^{j} v_{i j}=0, \quad j=1,2, \ldots, M_{i}$.

For example, if $k_{i} \neq k_{j}$ for $i \neq j$, then algebraic manipulation of Eq. (8) yields

$$
\begin{aligned}
C_{0}= & C_{0,0}+C_{N, 0}\left(1-\exp \left\{-k_{N} t\right\}\right), \quad N=2, \\
C_{0}= & C_{0,0}+C_{N, 0}\left(1-\exp \left\{-k_{N} t\right\}\right)+\sum_{i=2}^{N-1}\left(\alpha_{i}\left(1-\exp \left\{-k_{i} t\right\}\right)\right. \\
& \left.+k_{i} \sum_{j=i+1}^{N}\left(\frac{\alpha_{j} \prod_{m=i+1}^{j} k_{m}}{k_{j} \prod_{n=i}^{j-1}\left(k_{n}-k_{j}\right)}\right)\left(1-\exp \left\{-k_{j} t\right\}\right)\right), N \geqslant 3, \\
C_{1}= & C_{1,0}+C_{N, 0}\left(1-\exp \left\{-k_{N} t\right\}\right), \quad N=2, \\
C_{1}= & C_{1,0}+C_{N, 0}\left(1-\exp \left\{-k_{N} t\right\}\right)+\sum_{i=2}^{N-1}\left(C_{i, 0}-\alpha_{i} \exp \left\{-k_{i} t\right\}\right. \\
- & \left.\sum_{j=i+1}^{N}\left(\frac{\alpha_{j} \prod_{m=i+1}^{j}}{\prod_{n=i}^{j-1}\left(k_{n}-k_{j}\right)}\right) \exp \left\{-k_{j} t\right\}\right), N \geqslant 3, \\
C_{i}= & \alpha_{i} \exp \left\{-k_{i} t\right\}, i=N, N=2, \\
C_{i}= & \left.\left.\alpha_{i} \exp \left\{-k_{i} t\right\}+\sum_{j=i+1}^{N}\left(\frac{\alpha_{j} \prod_{m=i+1}^{j}}{\prod_{n=i}^{j-1}\left(k_{n}-k_{j}\right)}\right) \exp \left\{-k_{j} t\right\}\right)\right), \\
& 2 \leqslant i \leqslant N-1, N \geqslant 3, \\
C_{N}= & C_{N, 0} \exp \left\{-k_{N} t\right\}, \quad N \geqslant 2 .
\end{aligned}
$$

Each arbitrary constant $\alpha_{i}$ may be eliminated using the following recursive relation derived from the initial condition included in Eq. (8):

$$
\begin{aligned}
& \alpha_{N}=C_{N, 0}, \quad N \geqslant 2, \\
& \alpha_{i}=C_{i, 0}, \quad i=2, \quad N=2, \\
& \alpha_{i} \equiv C_{i, 0}-\sum_{j=i+1}^{N} \alpha_{j}\left(\frac{\prod_{m=i+1}^{j} k_{m}}{\prod_{n=i}^{j-1}\left(k_{n}-k_{j}\right)}\right), \quad 2 \leqslant i \leqslant N-1, \quad N \geqslant 3 .
\end{aligned}
$$

Although Eqs. (11)-(12) are involved especially when $N$ is large, a much simpler relationship is obtained if one assumes that $k_{i}=k_{j}=k$ for every $i \neq j$; in this situation, Eq. (8) can be rearranged to read

$$
\begin{aligned}
& C_{0}=C_{0,0}+\sum_{i=2}^{N} \int_{0}^{k t} \exp \{-k t\} \sum_{j=i}^{N} \frac{C_{j, 0}}{(j-i) !}(k t)^{j-i} \mathrm{~d}(k t), \quad N \geqslant 2, \\
& C_{1}=\left(\sum_{i=1}^{N} C_{i, 0}\right)-\exp \{-k t\} \sum_{j=2}^{N} C_{j, 0} \sum_{m=0}^{j-2} \frac{(k t)^{m}}{m !}, \quad N \geqslant 2, \\
& C_{i}=\exp \{-k t\} \sum_{j=i}^{N} \frac{C_{j, 0}}{(j-i) !}(k t)^{j-i}, \quad 2 \leqslant i \leqslant N, \quad N \geqslant 2 .
\end{aligned}
$$

Assuming in addition that all true reactants were initially present at the same concentration (i.e. $C_{0,0}=C_{1,0}=C_{2,0}=$ $\left.\ldots=C_{N, 0}\right)$, then Eq. (13) reduces to [8]

$$
\begin{aligned}
C_{0}= & C_{0,0}+(1+(N-1)(1-\exp \{-k t\})), \quad N=2, \\
C_{0}= & C_{0,0}(1+(N-1)(1-\exp \{-k t\}) \\
& +\sum_{j=2}^{N-1} \sum_{j=i+1}^{N}\left(1-\exp \{-k t\}\left(\frac{(k t)^{j-i}}{(j-i) !}\right.\right. \\
& \left.\left.\left.+\sum_{m=1}^{j-i} \frac{(k t)^{j-i-m}}{(j-i-m) !}\right)\right)\right), \quad N \geqslant 3,
\end{aligned}
$$

$C_{1}=C_{0,0}\left(N-\exp \{-k t\} \sum_{j=2}^{N} \sum_{m=0}^{j-2} \frac{(k t)^{m}}{m !}\right), \quad N \geqslant 2$,

$C_{i}=C_{0,0} \exp \{-k t\} \sum_{j=i}^{N} \frac{(k t)^{j-i}}{(j-i) !}, \quad 2 \leqslant i \leqslant N, N \geqslant 2$.

The variation of the various normalized concentrations with $k t$ for $N=6$ is highlighted in Fig. 1 .

If all polymeric substrates labile to enzyme action, i.e. $\mathrm{S}_{2}$, $\mathrm{S}_{3}, \ldots, \mathrm{S}_{N}$, were lumped together in a single, pseudo substrate $S$, the the overall reaction mechanism would simply be given by

$\mathrm{S}+\mathrm{E} \stackrel{K_{m}}{\rightleftarrows} \mathrm{E} . \mathrm{S} \stackrel{k_{\text {cat }}}{\longrightarrow} \mathrm{E}+\mathrm{S}_{1}+\mathrm{S}_{0}$

In this situation, the rate expression would read

$r=\frac{k_{c a t} C_{E, t o t} C}{K_{m}+C}$

where $C$ denotes the molar concentration of $S$, whereas the mass balances would be given by

$\frac{d\left(\begin{array}{c}C_{0} \\ C_{1} \\ C\end{array}\right)}{d t}=\left(\begin{array}{ccc}0 & 0 & k \\ 0 & 0 & k \\ 0 & 0 & -k\end{array}\right)\left(\begin{array}{l}C_{0} \\ C_{1} \\ C\end{array}\right)$,

$t=0,\left(\begin{array}{l}C_{0} \\ C_{I} \\ C\end{array}\right)=\left(\begin{array}{c}C_{0,0} \\ C_{1,0} \\ C_{0}\end{array}\right)$.

Integration of Eq. (17) for the case of $C_{0,0}=C_{1,0}$ and $C_{0}=(N-1) C_{0,0}$ would yield

$C_{0}=C_{0,0}(1+(N-1)(1-\exp \{-k t\}))$,

$C_{1}=C_{0,0}(1+(N-1)(1-\exp \{-k t\}))$,

$C=(N-1) C_{0,0} \exp \{-k t\}$.

The variation of the normalized concentrations vs. the dimensionless time is depicted in Fig. 2.

Denoting as $t_{\text {lag }}$ the (constant) time interval required for cleaning, loading and unloading the batch reactor, the existence of a maximum value for the average rate of monomer production [i.e. $\left(C_{0}-C_{0,0}\right) /\left(t+t_{\text {lag }}\right)$ ] is apparent from inspection of either 


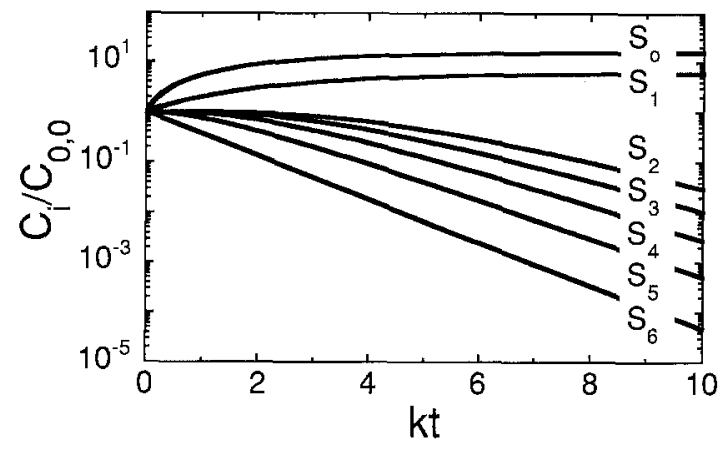

Fig. 1. Plots of the normalized concentrations of substrates $S_{0}$ through $S_{6}$ (i.e. $C_{0} / C_{0,0}$ through $C_{6} / C_{0,0}$ ) with the dimensionless batch time, $k t$, using the multisubstrate model

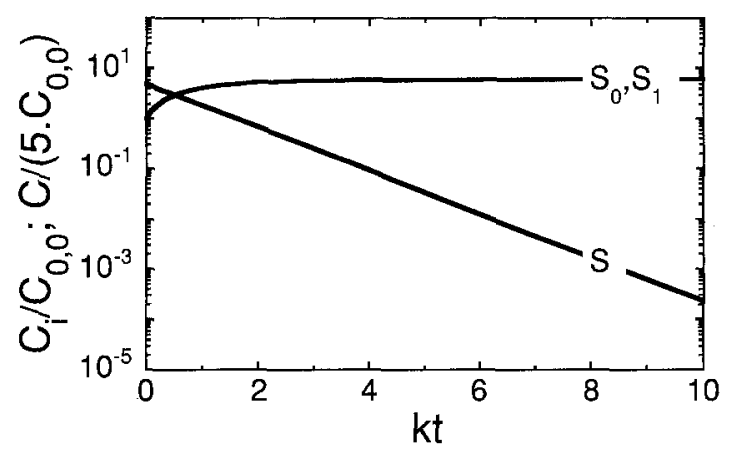

Fig. 2. Plots of the normalized concentrations of substrates $S_{0}$ and $S_{1}$ and lumped substrate $S$ [i.e. $C_{0} / C_{0,0}$ through $C_{1} / C_{0,0}$, and $\left.C /(N-1) C_{0,0}\right)$, with $N=6$ ] with the dimensionless batch time, $k t$, using the lumped substrate model

Fig. 3, which uses the true multisubstrate kinetic model, or Fig. 4, which uses the approximate lumped substrate kinetic model. The optimum operating batch time, $t_{\mathrm{opt}}$ (i.e. the time interval which leads to the maximum average rate of monomer production), can, thus, be obtained through

$$
\frac{\mathrm{d}\left(\frac{\frac{C_{0}}{C_{0,0}}-1}{k\left(t+t_{\mathrm{lag}}\right)}\right)}{\mathrm{d}(k t)}=0
$$

Combination of Eq. (14) with Eq. (19) yields, upon algebraic rearrangement $[8]$,

$k t_{\text {lag }}=\frac{1-\exp \left\{-k t_{o p t}\right\}}{\exp \left\{-k t_{o p t}\right\}}-k t_{\text {opt }}, \quad N=2$,

$k t_{\mathrm{lag}}=\frac{\left((N-1)\left(N+1-\exp \left\{-k t_{o p t}\right\}\right)-\frac{N(N+1)}{2}+1-\exp \left\{-k t_{\mathrm{opt}}\right\} \sum_{i=2}^{N-1} \sum_{j=i+1}^{N}\left(\frac{\left(k t_{\text {opt }}\right)^{j-i}}{(j-i) !}+\sum_{m=1}^{j-i} \frac{\left(k t_{o p t}\right)^{j-i-m}}{(j-i-m) !}\right)\right)}{\exp \left\{-k t_{\mathrm{opt}}\right\}\left(N-1+\sum_{i=2}^{N-1} \sum_{j=i+1}^{N} \frac{\left(k t_{o p t}\right)^{j-i}}{(j-i) !}\right)}$

$-k t_{\text {opt }}, \quad N \geqslant 3$,

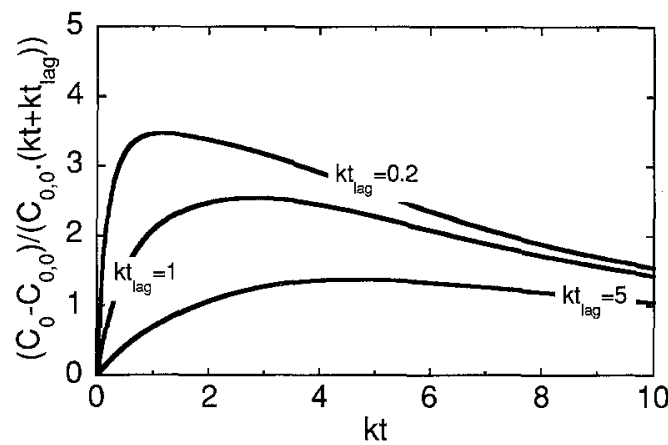

Fig. 3. Plot of the average dimensionless rate of production of monomer $S_{0}$ [i.e. $\left.\left(C_{0}-C_{0,0} / C_{0}\left(k t+k t_{\text {lag }}\right)\right)\right]$ with the dimensionless batch time, $k t$, for various values of the dimensionless lag time, $k t_{\text {lag }}$, using the multisubstrate model

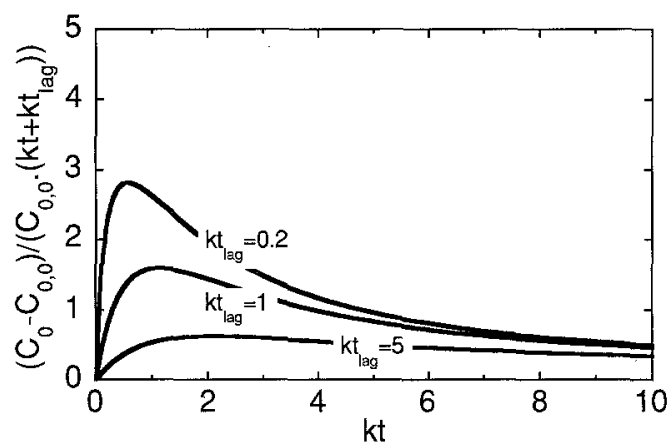

Fig. 4. Plot of the average dimensionless rate of production of monomer $S_{0}$ [i.e. $\left.\left(C_{0}-C_{0,0}\right) /\left(C_{0}\left(k t+k t_{\text {lag }}\right)\right)\right]$ with the dimensionless batch time, $k t$ for various values of the dimensionless lag time, $k t_{\text {lag }}$, using the lumped substrate model

which can be further simplified to give

$$
\begin{aligned}
& =\frac{N(N-1) \exp \left\{k t_{o p t}\right\}-\sum_{i=0}^{N-2} \frac{(N-i)(N-i-1)}{i !}\left(k t_{o p t}\right)^{i}}{2 \sum_{j=0}^{N-2} \frac{N-j-1}{j !}\left(k t_{o p t}\right)^{j}} \\
& -k t_{o p t}, \quad N \geqslant 2,
\end{aligned}
$$

where advantage was taken from the properties of the arithmetic series therein. Plots of the dimensionless optimum batch time, $k t_{o p t}$, versus the dimensionless lag time, $k t_{\text {lag }}$, using the multisubstrate model are depicted in Fig. 5 for several values of $N$. The corresponding maximum value for the average dimensionless rate of production of monomer, $r_{\mathrm{opt}}^{\star}$, may then be 


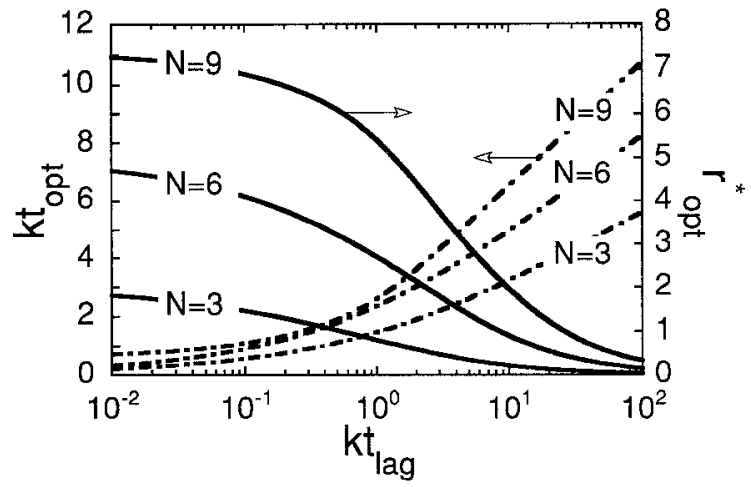

Fig. 5. Plot of the dimensionless optimum batch time, $k t_{o p t}$, and the dimensionless average rate of production of monomer $S_{0}, r_{o p t}^{*}$ (for $N=3$, $N=6$, and $N=9$ ), versus the dimensionless lag time, $k t_{\text {lag }}$, using the multisubstrate model

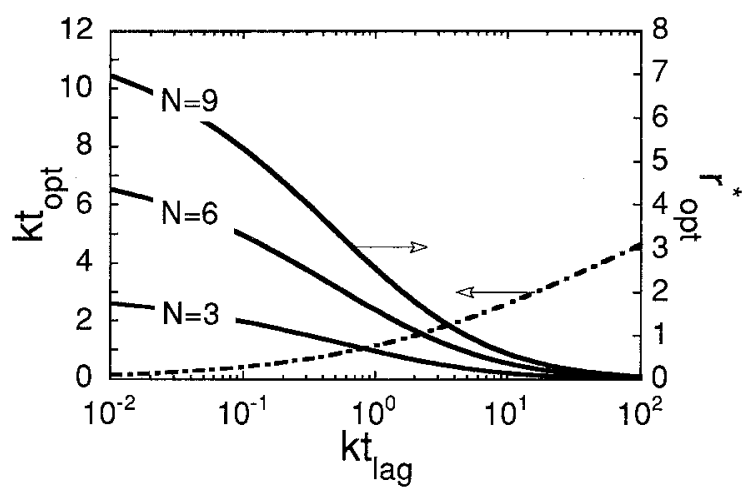

Fig. 6. Plot of the dimensionless optimum batch time, $k t_{\text {opt }}$ ( for $N=3, N=6$, and $N=9$ ), and the dimensionless average rate of production of monomer $S_{0}$, $r_{\text {opt }}^{*}$ (for $N=3, N=6$, and $N=9$ ), versus the dimensionless lag time, $k t_{\text {lag }}$, using the lumped substrate model

obtained via:

$r_{o p t}^{*}=\frac{\frac{C_{0}\left\{t=t_{o p t}\right\}}{C_{0,0}}-1}{k t_{o p t}+k t_{\text {lag }}}=\exp \left\{-k t_{\text {opt }}\right\}\left(\sum_{i=0}^{N-2} \frac{N-i-1}{i !}\left(k t_{\text {opt }}\right)^{i}\right)$,

$$
N \geqslant 2 \text {, }
$$

which resulted from Eq. (22). Plots of $r_{o p t}^{*}$ versus the dimensionless lag time, $k t_{\text {lag }}$, using the multisubstrate model are also depicted in Fig. 5 for several values of $N$.

Combination of Eqs. (18)-(19) yields

$k t_{\text {lag }}=\exp \left\{k t_{o p t}\right\}-1-k t_{\text {opt }}$.

Plots of $k t_{o p t}$ vs. $k t_{\text {lag }}$ using the lumped substrate approach are available in Fig. 6. In a similar way as before, the corresponding maximum value for the average dimensionless rate of production of monomer, $r_{o p t}^{*}$, may be obtained via

$r_{o p t}^{*} \equiv \frac{\frac{C_{0}\left\{t=t_{o p t}\right\}}{C_{0,0}}-1}{k t_{o p t}+k t_{\text {lag }}}=(N-1) \exp \left\{-k t_{o p t}\right\}$,

which was, in turn, obtained from Eq. (23). Plots of $r_{\text {opt }}^{\star}$ versus the dimensionless lag time, $k t_{\text {lag }}$, using the lumped substrate model are also depicted in Fig. 6 for several values of $N$.

\section{2}

\section{Discussion and conclusions}

The general shape of the curves representing the variation of the dimensionless concentrations of the various substrates for a common initial concentration has one of two distinct behaviors: either (i) the concentration is a monotonically increasing function of time, which is the case of $C_{0}$ and $C_{1}$, the values of which are a direct result of the decrease in concentration of all heavier substrate species; or (iii) the concentration is a monotonically decreasing function of time, which is the case of $C_{2}, C_{3}, \ldots, C_{N}$, each of which is described by the product of a monotonically increasing polynomial in time by a monotonically describing exponential of time, where the latter decreases faster than does the former, and tends to an asymptotic behavior for large times given by [recall Eq. (13)]

$\lim _{k t \rightarrow \infty} C_{i}=\frac{(k t)^{N-i}}{(N-i) !} \exp \{-k t\}$,

which gives rise to the approximately linear behavior of the logarithmic plots which is apparent in Fig. 1 (remember that the increase in $\log (k t)$ is much slower than the increase in $k t$ itself for large $k t$ ) with a slope of negative unity.

Based on inspection of Figs. 5 and 6, one concludes that the loci of the maxima for the average rate of monomer production decrease with the lag time whereas the loci of the corresponding optimum times increase with the lag time (also cf. Figs. 3 and 4). This means that, as expected, the amplitude of the lag time plays a crucial role in the optimization of the operation of the batch enzyme reactor. For longer $k t_{\text {lag }}$ it is also observed that $k t_{\text {opt }}$ becomes an essentially linear function in $k t_{\text {lag }}$, with slope depending on the value of $N$. In addition, an increase in the size of the largest polymer (i.e. an increase in $N$ ) leads to increases in both $k t_{o p t}$ and $r_{o p t}^{\star}$ for a given $k t_{\text {lagi }}$, hence, the overall production of monomer is accomplished in a better way if the monomer is released from substrate molecules with a wide range of sizes instead of being released from substrate molecules with the same (given) size and initial concentration equal to the sum of initial concentrations of all substrates in the former situation. It is interesting to note that $k t_{\text {opt }}$ is the same for a given $k t_{\text {lag }}$ irrespective of $N$ if the lumped substrate approach is employed, although this fact does not hold for $r_{o p t}^{*}$.

The variation of $r_{\text {opt }}^{*}$ with $k t_{\text {lag }}$ is actually more damped (cf. Fig. 5) than would have been predicted on the basis of the lumped substrate approach (cf. Fig. 6), and the difference between the two predicted behaviors is largest at lag times in the order of $1 / k$. Furthermore, the true $k t_{\text {opt }}$ is always above the hypothetical $k t_{\text {opt }}$ if the lumped model were valid. Therefore, using the lumped approximation would consistently indicate that the batch reactor should be stopped before the true optimum, thus giving rise to a nonconservative decision (the inadequacy of the operation pattern based on the lumped model worsens as $N$ increases). The aforementioned rationale corroborates previous works [9] on the suitability of simplistic lumped substrate approximations in multisubstrate reaction systems. 
This communication serves the practical purpose of providing simplified analytical criteria in dimensionless form able to support optimal operation (from an engineering point of view) of biochemical batch reactors, which are particularly relevant for operation on an industrial scale. Even if the various $k_{i}$ and $k_{-i}$ values are different from one another (as in the most general situation), the simple results outlined in Eqs. (22) and (23) still allow a better prediction of the best operating conditions than the lumped substrate approach highlighted in Eqs. (24) and (25); in this respect, the reasoning developed here serves the useful goal of providing a simple overview of the effect of multiple substrates in the optimal behavior of a batch reaction system.

\section{References}

1. Marison, I. W.: Enzyme kinetics. In: Scragg, A. H. (Ed.), Biotechnology for engineers - biological systems in technological processes. p. 96, Ellis Horwood, Chichester (1988)

2. Bailey, J. E.; Ollis, D. F.: Biochemical Engineering Fundamentals, McGraw-Hill, New York (1986)

3. Chipman, D. M.: Biochemistry 10 (1971), 1714

4. Hiromi, K.; Ogawa, K.; Nakanishi, N.; Ono, S.: J. Biochem. 60 (1966) 439

5. Ono, S.; Hiromi, K.; Zinbo, M.: J. Biochem. 55 (1964) 315

6. Malcata, F. X.: Int. J. Math. Ed. Sci. Technol. (1993) [in press]

7. Braun, M.: Differential equations and their applications - an introduction to applied mathematics, Springer, New York (1978)

8. Gradshteyn, I. S.; Ryzhik, I. M.: Table of Integrals, Series, and Products, Academic Press, San Diego (1980)

9. Malcata, F. X.: J. Chem. Ed. 68 (1991) 288 